\title{
A Simple and Fast Computation Equivalent Circuit Model to Investigate the Effect of Tape Twisting on the AC Loss of HTS Cables
}

\author{
Alireza Sadeghi \\ Department of Electrical Engineering \\ Shahrood University of Technology \\ Shahrood, Iran \\ alisadeghi380@yahoo.com
}

\author{
Seyyed Mesysam Seyyed Barzegar \\ Department of Electrical Engineering \\ Shahrood University of Technology \\ Shahrood, Iran \\ seyyedbarzegar@shahroodut.ac.ir
}

\author{
Mohammad Yazdani-Asrami \\ James Watt School of Engineering \\ University of Glasgow \\ Glasgow, UK \\ m.yazdaniasrami@gmail.com
}

\begin{abstract}
This paper aims to evaluate the AC loss of a High Temperature Superconducting (HTS) cable with respect to the twisting angle while considering mechanical constraints in an iterative approach. A $1 \mathrm{~km} 22.9 \mathrm{kV}$ AC HTS cable was selected in this study to assess the impact of the twisting angle alterations. The electromagnetic behavior of the selected HTS cable was modeled using an Equivalent Circuit Model (ECM). After the implementation of this model in MATLAB/SIMULINK, a series of simulations were performed without the consideration of mechanical limits. They showed that the increase in the twisting angle leads to the decrease of the AC loss. Afterwards, simulations were conducted to reduce the AC loss, while mechanical limits were taken into account. This could reduce the AC loss by $27.41 \%$ with a much lower computation time than Finite Element Methods (FEMs).
\end{abstract}

Keywords-HTS cables; power cables; twisting; magnetic field; AC loss

\section{INTRODUCTION}

Since the discovery of superconductors in 1911 by H. K. Onnes, many investigations were initiated to enhance the critical temperature of superconductors. In 1986, J. G. Bednorz and K. A. Müller have introduced new superconductors with a critical temperature near $30 \mathrm{~K}[1]$. This has led to the rapid development in the manufacturing of High Temperature Superconducting (HTS) tapes and wires. Ever since the application of superconductors in power systems is gaining interest. Consequently, numerous investigations were funded to take the advantage of HTS materials in various apparatuses of power systems [2-9]. Among them, HTS cables are the most investigated components. They can be applied in common power systems as transmission or distribution lines [7]. They also play a significant role in future power systems with renewable energy resources and even smart grids [10-14]. Accordingly, multiple studies have dedicated their efforts and funding to improve and model the electromagnetic and thermomechanical characteristics of HTS cables. Altogether, the main objective of these studies is to attain the proper behavior of HTS cables and increase their efficiency. One of the most significant parameters which could entirely impact the characteristic of cables is AC loss. Along with the passing of an AC current through HTS tapes of a superconducting cable, alternating magnetic fields are generated in the HTS cable [15]. The AC current and the generated fields induce losses in HTS cables which are known as transport current loss and hysteresis loss respectively [16]. These losses increase the heat load of the cooling system and consequently, the efficiency of the cable is reduced $[17,18]$. To avoid such consequences, numerous attempts were conducted to reduce the AC loss of cables.

In [19] the AC loss of a $66 \mathrm{kV}$ HTS cable was studied and reduced by the variation in the topology of the HTS cable. This change is based on the increase of the radius of the former. By doing this, the number of HTS tapes is increased in each layer and a lower current passes through each tape. However, this method increases the total cost and weight of the cable. In [20], the AC loss is reduced by the reduction of tape thickness. The thickness of tapes directly impacts their engineering current density and so, AC loss. A sensitivity analysis was conducted in [21] to reduce the AC loss of the cable by the variation of the twisting angle. This method is very effective and can reduce AC loss significantly. Nonetheless, there is a lack of an iterative algorithm in this method to guarantee the certainty of the results. Authors of [22] have also used the twisting angle increase to suppress the AC loss. However, they did not consider any types of mechanical constrain on the HTS tapes. Twisting angle was also used for the sake of gaining a uniform 
current distribution in HTS cables, without any attention to AC loss $[23,24]$. The novelty of this paper is the reduction of the $\mathrm{AC}$ loss by varying the twisting angle in an iterative method while the mechanical limits of the HTS tapes are considered to avoid mechanical issues. A $22.9 \mathrm{kV}$ HTS cable with a length of $1 \mathrm{~km}$ is opted to apply variations of twisting angle and the design improvement. All simulations were carried out in MATLAB/SIMULINK. The results show that the twisting angle has a significant impact on the value of the AC loss.

\section{StRUCtURE OF THE HTS CABLE AND SIMULATION PROCEDURE}

\section{A. Description of the HTS Cable and ECM}

To analyze the proposed method, a $22.9 \mathrm{kV}$ coaxial HTS cable was considered [25]. Figure 1(a) illustrates the structure of the chosen cable and Table I tabulates the most important parameters of the cable. According to Figure 1(a), the superconducting tapes of the cable are 2G HTS tapes. More detailed information about these tapes is listed in Table II. The selected HTS cable is a connection between the $22.9 \mathrm{kV}$ generation units and a 50 MVA load at $50 \mathrm{~Hz}$ frequency. In this study, an ECM is put to work to simulate the behavior of the selected HTS cable.

(a)

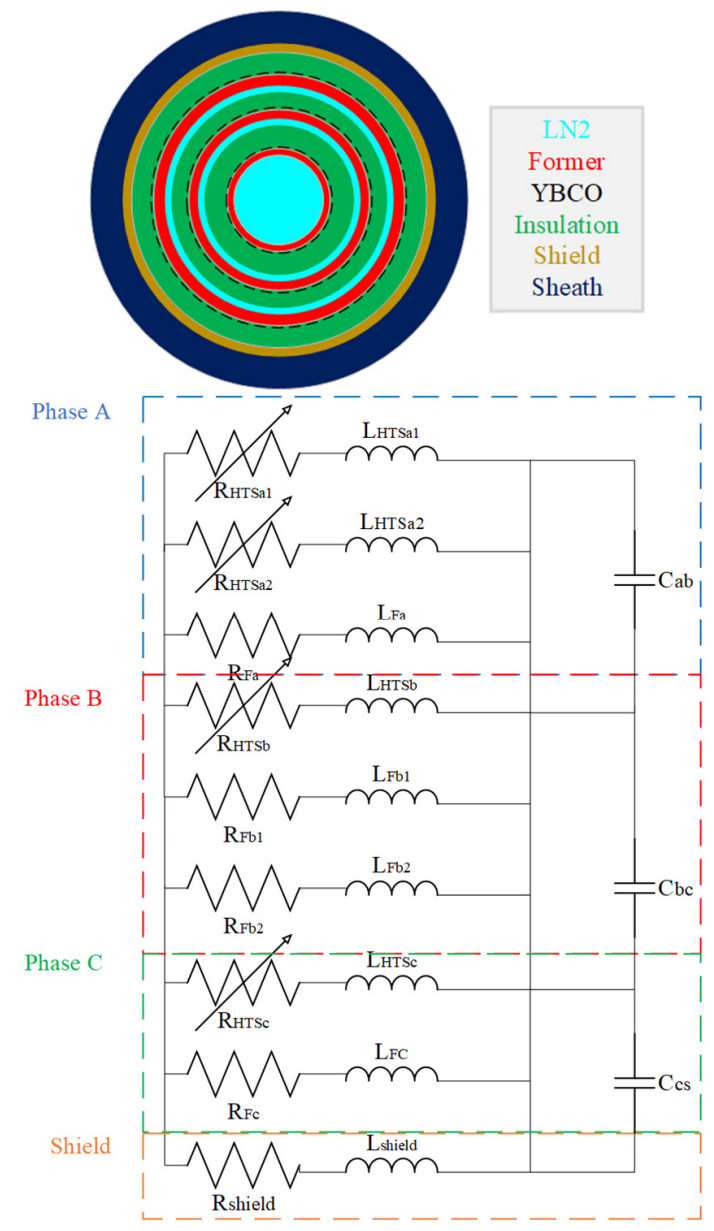

Fig. 1. The structure of (a) HTS cable, (b) equivalent circuit model of the understudied cable.
Compared to FEMs, ECMs not only have a lower simulation time but also can provide a more complex test power system with different buses, lines, and generation units for the simulation of an HTS cable. So, an ECM for $22.9 \mathrm{kV}$ HTS cable was provided which is shown in Figure 1(b). In this Figure, the subscript "HTS" is devoted to the resistances and inductances of superconducting layers in each phase while " $F$ " is related to former layers. There are also mutual inductances which are not shown in Figure 1(b) to avoid complexity. However, self-inductances, mutual-inductance, and capacitances were calculated according to [26].

TABLE I. INFORMATION OF THE SELECTED HTS CABLE

\begin{tabular}{|c|c|}
\hline Item & Value and Type \\
\hline Voltage/Power (kV/MVA) & $22.9 / 50$ \\
\hline Tape & YBCO \\
\hline Operational Temperature (K) & 70 \\
\hline Insulation Layer & PPLP \\
\hline Primary pitch angle in each phase [A1 & {$\left[18.1^{\mathrm{O}}-16.3^{\mathrm{O}} 30.7^{\mathrm{O}} 45.8^{\mathrm{O}}\right]$} \\
\hline A2 B C] & {$[17202128]$} \\
\hline Former Radiuses [A B1 B2 C] $(\mathrm{mm})$ & LN2 \\
\hline Coolant & \\
\hline
\end{tabular}

TABLE II. YBCO TAPE CHARACTERISTICS

\begin{tabular}{|c|c|}
\hline Parameter & Value \\
\hline Tape Type & YBCO \\
\hline Width and Thickness $(\mathrm{mm})$ & $4.4 \& 0.1$ \\
\hline Stabilizer Thickness & $\begin{array}{c}50 \text { (25 upper stabilizer and } \\
25 \text { lower stabilizer })\end{array}$ \\
\hline Upper Shield Thickness $(\mu \mathrm{m})$ & 2 \\
\hline Lower Shield Thickness $(\mu \mathrm{m})$ & 1.8 \\
\hline YBCO Thickness $(\mu \mathrm{m})$ & 1 \\
\hline Substrate Thickness $(\mu \mathrm{m})$ & 45.2 \\
\hline
\end{tabular}

\section{B. Modeling Formulation}

The passing current through the cable induces a magnetic field with perpendicular and parallel components. These components vary with respect to the twisting angle and the pitch length. According to Biot-Savart law, these components of the magnetic fields are computed as follows [27]:

$$
\begin{aligned}
& B_{i z}= \mu_{0}\left(\sum_{k=i-1}^{n} a_{k} \frac{I_{k}}{l_{p k}}+a_{i}\left(\frac{r_{i o}-r_{i p}}{r_{i o}-r_{i i}}\right) \frac{I_{i}}{l_{p i}}\right) \\
& B_{i \theta}= \mu_{0}\left(\frac{1}{2 \pi r_{i p}} \sum_{k=i}^{i-1} I_{k}+\left(\frac{r_{i p}-r_{i i}}{r_{i o}-r_{i i}}\right) \frac{I_{i}}{2 \pi r_{i o}}\right) \\
& B_{i \perp}=B_{i z} \cos \alpha_{i}+B_{i \theta} \sin \alpha_{i} \\
& B_{i \|}=B_{i z} \sin \alpha_{i}+B_{i \theta} \cos \alpha_{i}
\end{aligned}
$$

where $B_{i \perp}$ is the perpendicular component of the magnetic field in ith layer and $B_{i \|}$ is the parallel magnetic field, $\alpha_{i}$ is the twisting angle, $B_{i z}$ states the axial magnetic field, and $B_{i \theta}$ expresses the azimuthal magnetic field.

Equations (5-8) represent the formulations of the pitch length in each layer of the cable [28]:

$$
\begin{gathered}
\ell_{a, 1}=\varepsilon f_{1}\left(r_{a}, r_{b}, r_{c}\right) \\
\ell_{a, 2}=-\varepsilon f_{1}\left(r_{a}, r_{b}, r_{c}\right) \\
\ell_{b, 1}=\varepsilon f_{2}\left(r_{a}, r_{b}, r_{c}\right)
\end{gathered}
$$




$$
\ell_{c, 1}=\varepsilon f_{3}\left(r_{a}, r_{b}, r_{c}\right)
$$

where $\ell_{a b c, i}$ is the primary twisting pitch of th $i^{\text {th }}$ layer of the three phases and $r_{a b c}$ is the radii of the formers in each phase. As it can be seen, the twisting length is a function of the former radius. By accessing the pitch length of the phases, the calculation of the primary pitch angle of the HTS cable is conductible according to (9) [29]:

$$
\alpha=\tan ^{-1} \frac{\ell_{i}}{2 \pi r_{i}}
$$

The calculated twisting length and the twisting angle of the understudied cable based on (5-9) are tabulated in Table I as simulation initial values. By having the magnetic field and the current distribution in each tape, the $\mathrm{AC}$ loss could be attained according to [30-32]:

$$
\begin{gathered}
Q_{\text {mag }}=\left\{\begin{array}{cc}
\frac{2 f B_{i}{ }^{2}}{\mu_{0}} \frac{\beta_{i}}{3} S_{i} & \beta_{i}<1 \\
\frac{2 f B_{i}{ }^{2}}{\mu_{0}}\left(\frac{1}{\beta_{i}}-\frac{2}{3 \beta_{i}{ }^{3}}\right) S_{i} & \beta_{i}>1
\end{array}\right\} \quad(10) \\
B_{i}=\sqrt{{B_{i z}}^{2}+B_{i \theta}{ }^{2}} \quad(11) \\
\beta_{i}=\frac{B_{i}}{\mu_{0} J_{C} b} \quad(12) \\
Q_{\text {trans }}=\frac{\mu_{0} f I_{C i}{ }^{2}}{2 \pi}\left\{\left(2-F_{i}\right) F_{i}+2\left(1-F_{i}\right) \ln \left(1-F_{i}\right)\right\} \\
F_{i}=\frac{I_{P i}}{I_{C i}} \quad(14)
\end{gathered}
$$

where $Q_{\text {mag }}$ is the magnetization loss in tapes, $Q_{\text {trans }}$ is the transport current loss of superconducting layers, $f$ is the frequency, $\mu_{0}$ is the permeability of vacuum, $S_{i}$ is a crosssection of layer $i, J_{C}$ is critical current density, $b$ is the half width of HTS tapes, $I_{C i}$ is critical current of the $i^{\text {th }}$ layer, and $I_{P i}$ is the peak value of the current.

According to (10-14), twisting angle has a remarkable impact on the magnetic field and on AC loss [33]. As a matter of fact, the AC loss of a superconducting cable could be reduced by changing the initially calculated twisting angles. However, changing the twisting angle could jeopardize the mechanical stability of the tapes and this could lead to malfunctions in the HTS cable. To attain the mechanical limits, firstly strain must be calculated. The strain is a function of twisting pitch and the calculation point of strain. This could be conducted as expressed in (15). If the mechanical limits surpass the threshold value, the critical current abruptly becomes zero. The correlation between the strain and the critical current of tapes is expressed in (15-19) [34]:

$$
\begin{aligned}
& \varepsilon(d, \ell)=2 \pi^{2} \frac{\left(d^{2}-\frac{\omega^{2}}{12}\right)}{\ell^{2}} \\
& I_{c}(T, \varepsilon)=I_{c m}(T)\left[1-b(T)\left|\varepsilon-\varepsilon_{m}(T)\right|^{2}\right] \quad \forall \varepsilon \leq \varepsilon_{i r r} \\
& I_{c}(T, \varepsilon)=0 \quad \forall \varepsilon>\varepsilon_{\text {irr }} \\
& b(T)=b_{0}+\operatorname{dexp}(e T) \\
& \varepsilon_{m}(T)=\mathrm{F} T-\varepsilon_{m_{0}} \\
& I_{c m}(T)=I_{c_{m 0}}\left(1-\frac{T}{T_{C_{1}}}\right)^{m}
\end{aligned}
$$

In (16), $\varepsilon_{i r r}$ is the maximum strain value of tapes. The other parameters are tabulated in Table III [34].

TABLE III. PARAMETERS OF (16-19)

\begin{tabular}{|c|c|}
\hline Parameter & Value \\
\hline $\boldsymbol{I}_{\boldsymbol{c}_{\boldsymbol{m} \mathbf{0}}}$ & 1282 \\
\hline $\boldsymbol{T}_{\boldsymbol{C}_{\mathbf{1}}}$ & 94 \\
\hline $\boldsymbol{M}$ & 1.45 \\
\hline $\boldsymbol{b}_{\boldsymbol{0}}$ & 3122 \\
\hline $\boldsymbol{d}$ & $4.3 \mathrm{e}-4$ \\
\hline $\boldsymbol{e}$ & 0.201 \\
\hline $\boldsymbol{\varepsilon}_{\boldsymbol{m}_{\mathbf{0}}}$ & 0.44 \\
\hline $\boldsymbol{F}$ & $5.1 \mathrm{e}-5$ \\
\hline $\boldsymbol{\varepsilon}_{\boldsymbol{i r r}}$ & 1.1 \\
\hline
\end{tabular}

Figure 2 illustrates the normalized value of the critical current in different locations while the strain is varied. As shown in Figure 2, the maximum value of strain in phase $A$ is about $0.5 \%$, while this value for phase $\mathrm{B}$ is about $0.95 \%$ and in phase, $\mathrm{C}$ is $1 \%$. So, the angle of phase $\mathrm{C}$ should not be increased any further or the mechanical collapse will occur in this phase. On the other hand, in phases $\mathrm{A}$ and $\mathrm{B}$, the maximum induced strain is lower than $\varepsilon_{i r r}$ and so, their angle can be changed. After analyzing the resulting critical current of different phases, it can be observed that the tapes of phase A are operating way lower than $\varepsilon_{i r r}$ and the angle of this phase could be further increased to reduce the AC loss of the system. The tapes of phase B have the maximum strain value of $0.95 \%$ and could be twisted a little further.

\section{Simulation Procedure of the Proposed Method}

To this section, the formulation, structure of the test system, the geometry of the HTS cable, and the structure of ECM are discussed. This section is presented to characterize the proposed modeling procedure and the $\mathrm{AC}$ loss reduction. Figure 3(a) represents the Simulink model of the test system. It can be seen that HTS cable with $1 \mathrm{~km}$ length is used to connect the upstream grid and the load. The HTS cable model is shown in Figure 3(b). Each phase of the cable is modeled by a variable resistance for HTS tapes, a constant resistance for the former layer, and a matrix of self and mutual inductances between all layers. Each variable resistance consists of multiple parallel superconducting tapes. One of these tapes is illustrated in Figure 3(c). In this Figure, RYBCO is the resistance of the superconducting layer of tape, RAg is the resistance of a silver layer of the tape, and $\mathrm{RCu}$ and Rsolder imply the resistivity of the copper stabilizer layer and solder, respectively. These resistances along with the resistivity of the former and the inductances are calculated in each iteration and inserted into the system. The procedure is shown in Figure 4. The first step is to feed the model with the former radii and to calculate the twisting characteristics as an initial value for the model. After that, the iteration number is set to 1 and after inserting the values of resistances and inductances, the Simulink model is run. After that, the strain for the calculated twisting angle is computed. In the next step, the computed strain is compared with the maximum irreversible strain of HTS tapes. If the strain is lower than this limit, a value as $\Delta \alpha$ is added to the last twisting angle and another time Simulink model is run. This procedure continues until the strain overpasses the irreversible 
strain limit and that results in stopping the algorithm. $\Delta \alpha$ plays an important role in the speed of the proposed method. The smaller this value is, the higher the computation time would be.

(a)

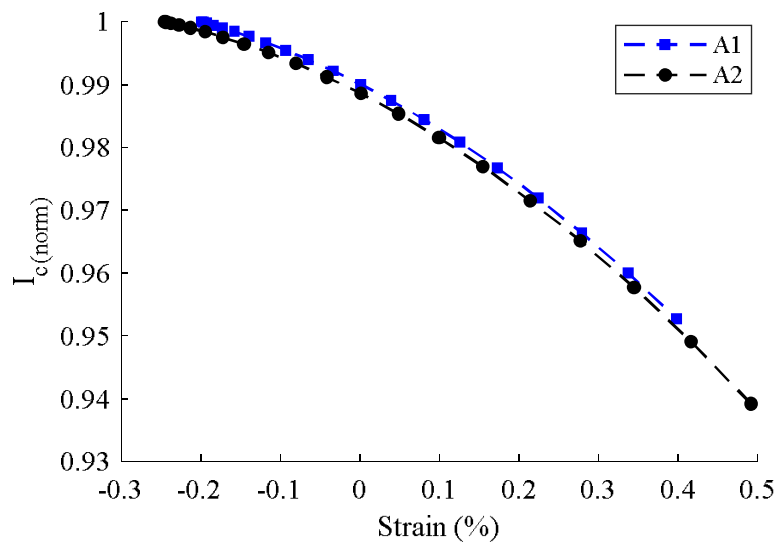

(b)

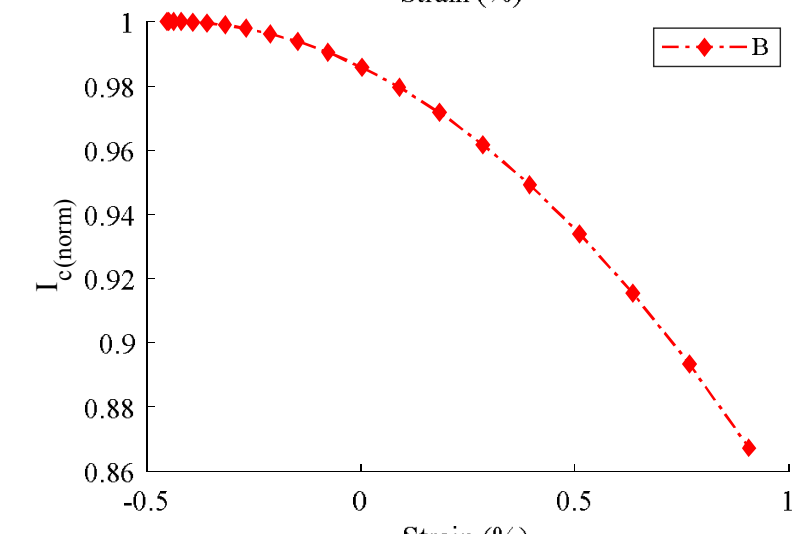

(c)

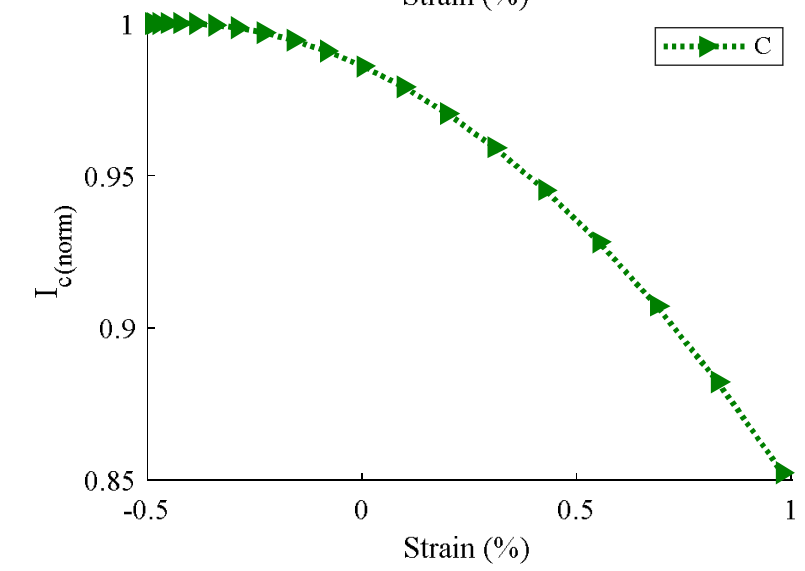

Fig. 2. Normalized critical current value with respect to strain for the primary angle of tapes: (a) of phase A, (b) of phase B, (c) of phase C.

(a)

Utility Grid

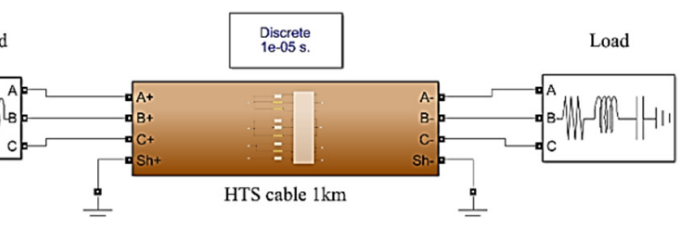

(b)

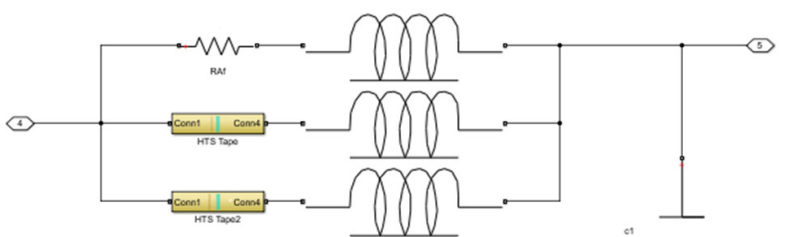

(c)

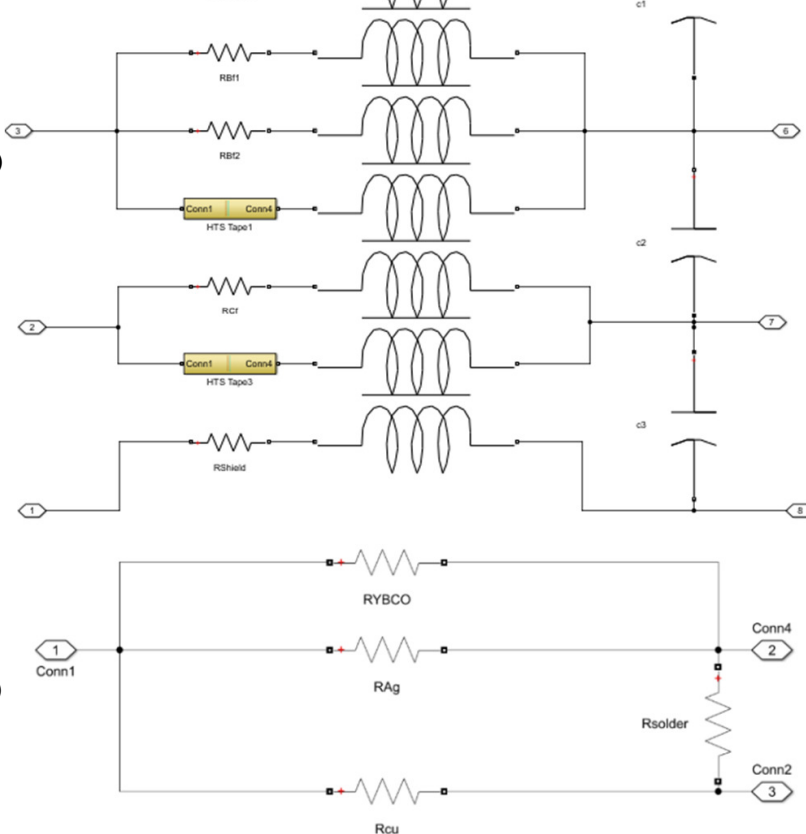

Fig. 3. Simulink model used as the test system of the proposed method: (a) system model, (b) cable model, (c) HTS tape model.

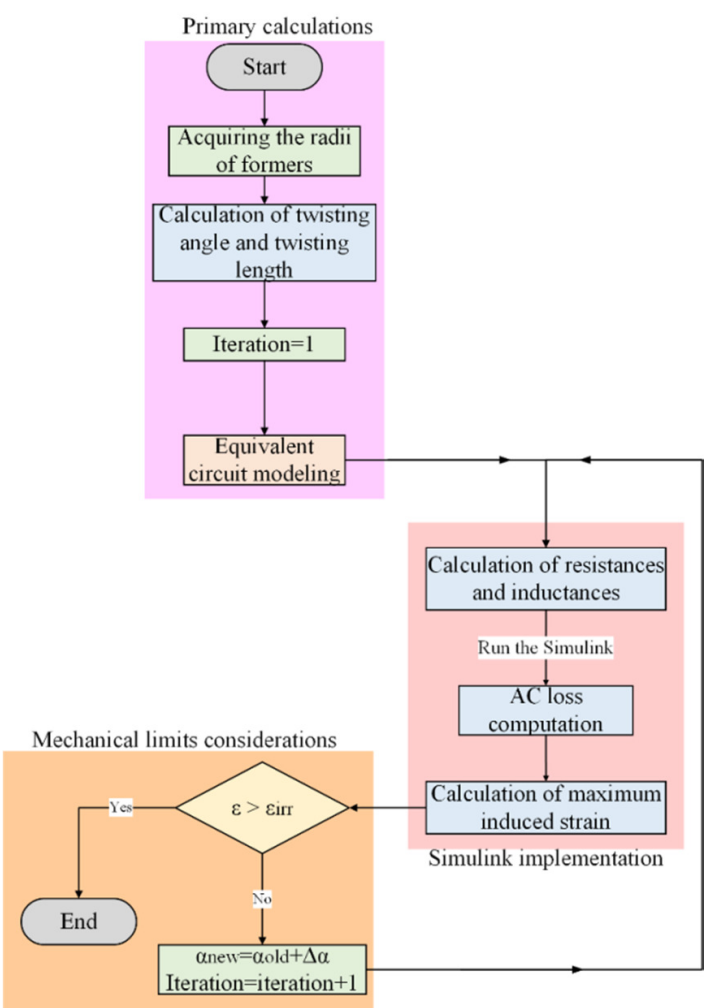

Fig. 4. The general flowchart for implementing the proposed method. 


\section{RESULTS AND DISCUSSION}

\section{A. Sensitivity Analysis of Twisting in an HTS Cable without Consideration of Mechanical Limits}

The twisting angle impacts the parallel and perpendicular components of the magnetic field. Figure 5 shows the changes of parallel and perpendicular components of the magnetic fields of each phase. According to Figure 5, the parallel component of the magnetic field is maximum for twisting angle of $0^{\circ}$, whereas the maximum perpendicular magnetic field occurs in $90^{\circ}$. The values of Figure 5 are calculated by neglecting the mechanical limits of the tapes.

(a)
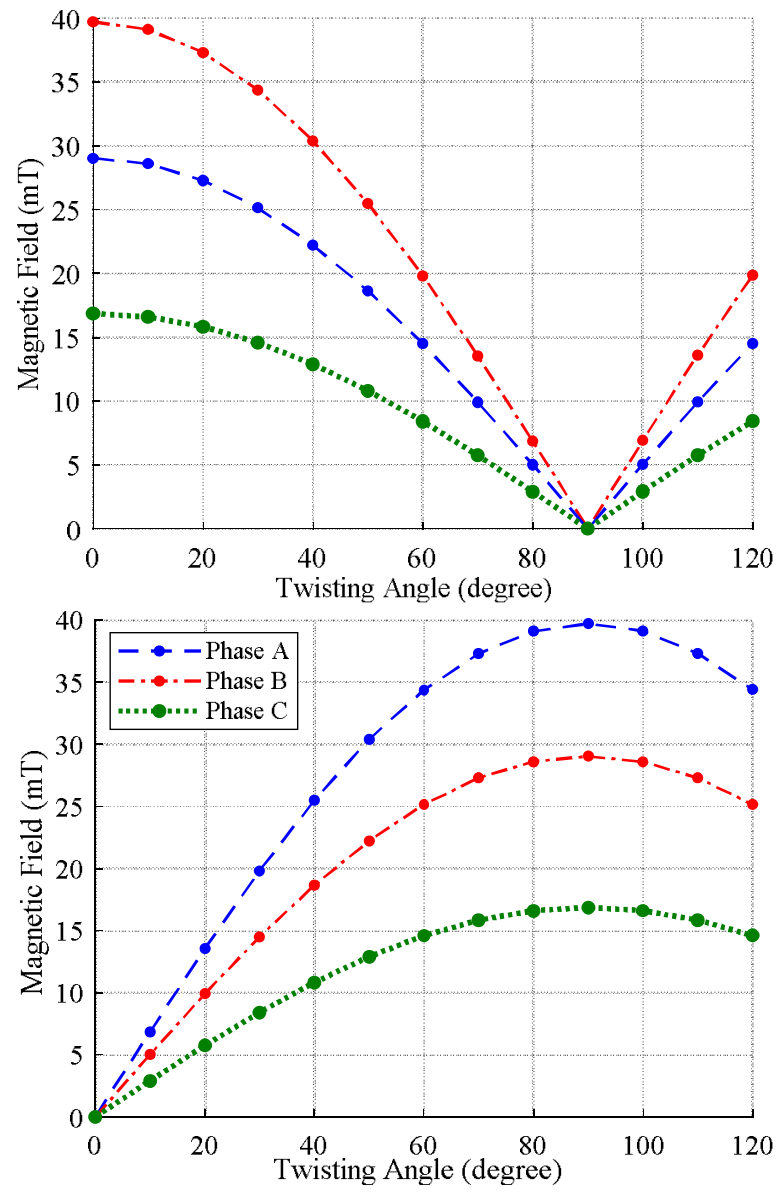

Fig. 5. (a) Parallel, (b) perpendicular magnetic field variation with respect to angle.

AC loss reduction is the most important target of this paper. $\mathrm{AC}$ loss is reduced by the increase in twisting angle with respect to parallel and perpendicular magnetic fields. Figure 6 illustrates the behavior of AC loss with respect to pitch angle alterations. According to Figure 6, a twisting angle of $90^{\circ}$ produces the minimum AC loss, near-zero, however, this led to the mechanical collapse of the tapes.

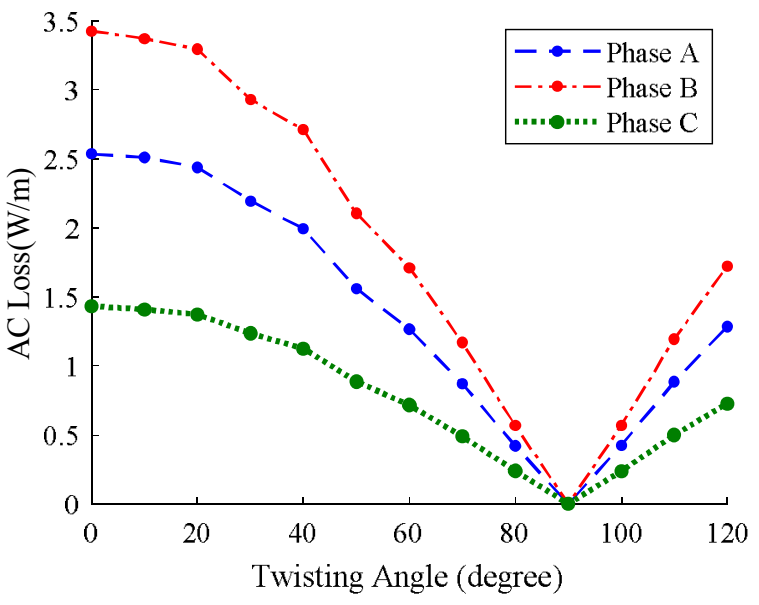

Fig. 6. $\mathrm{AC}$ loss variations with respect to twisting angle.

B. The Results of the Proposed Method in the Reduction of the AC Loss of the HTS Cable with Consideration of the Mechanical Limits

In order to reduce the AC loss of the HTS cable, the twisting angle of the HTS cable could undergo some changes. The proposed ECM in this paper can analyze the aforementioned electromechanical problems at a significantly faster time (than FEM) with high accuracy while the mechanical limits are considered. Firstly, the twisting angles of HTS tapes in phase A (two layers of tapes), phase B, and phase- $\mathrm{C}$ are increased according to the procedure of Figure 4 with a $\Delta \alpha$ step, and pitch lengths are recalculated. After gaining these parameters of the HTS cable, the strain is computed in each iteration to avoid the downgrading of the critical current to zero. The basic value of the AC loss without any changes in twisting angle is about $3.125 \mathrm{~W} / \mathrm{m} / \mathrm{ph}$. To reduce this value and change the twisting angle, multiple $\Delta \alpha$ are presented in Table IV. According to this Table, the smaller $\Delta \alpha$ leads to a more precise solution. However, the simulation time increases significantly. This means that for a $0.0644 \%$ further reduction in AC loss, computation time is 886.3333 times increased.

TABLE IV. ANGLE VARIATION IMPACT ON AC LOSS REDUCTION

\begin{tabular}{|c|c|c|c|c|}
\hline$\Delta \alpha$ (degrees) & 0.05 & 0.005 & 0.0005 & 0.00005 \\
\hline $\begin{array}{l}\text { Final } \alpha \text { ngle } \\
\text { (degrees) }\end{array}$ & 35.3612 & 35.2332 & 35.2292 & 35.2284 \\
\hline$\varepsilon(\%)$ & 1.105761 & 1.099339 & 1.099065 & 1.099059 \\
\hline$\frac{I_{C}(\varepsilon)}{I_{C}(0)}$ & 0.899 & 0.8988 & 0.8965 & 0.8963 \\
\hline $\begin{array}{c}\text { Maximum AC loss } \\
(W / m / p h)\end{array}$ & 2.25168 & 2.25043 & 2.25026 & 2.25023 \\
\hline Iteration & 159 & 1593 & 15927 & 159263 \\
\hline Time (s) & 0.03 & 0.32 & 2.72 & 26.59 \\
\hline Improvement (\%) & 27.9462 & 27.9862 & 27.9917 & 27.9926 \\
\hline
\end{tabular}

Table V shows the comparison results bewtween the proposed method and other previously published methods. The conducted studies in $[21,22]$ have also aimed to reduce the AC loss by the variation of the twisting angle. The maximum reduction of the AC loss in [21] is about $19.66 \%$ while the mechanical limits were also considered. However, the current paper uses sensitivity analysis to gain the results and does not 
present any iterative method. On the other hand, in [22] the AC loss is reduced by 12 to $14 \%$, but the mechanical limits were neglected.

TABLE V. COMPARISON BETWEEN THE PROPOSED METHOD AND $[21,22]$

\begin{tabular}{|c|c|c|c|}
\hline Reference & Maximum reduction & Mechanical limits & Iterative method \\
\hline$[\mathbf{2 1}]$ & $19.66 \%$ & yes & no \\
\hline$[\mathbf{2 2}]$ & $12-14 \%$ & no & no \\
\hline Proposed & $27.99 \%$ & yes & yes \\
\hline
\end{tabular}

\section{CONCLUSION}

$\mathrm{AC}$ loss is investigated in this paper, as a significant parameter in the performance of superconducting cables, with its reduction being the main target. This paper has utilized an ECM to analyze the impact of the twisting angle increment on the efficiency improvement of the selected HTS cable. For this purpose, firstly a sensitivity analysis was conducted to analyze the electromagnetic behavior of the cable which results in:

- The parallel magnetic field was reduced along with the increment of twisting angle, while the perpendicular magnetic field was increased.

- The AC loss of the cable in all phases was either decreased to a minimum value for the angle of about 90 degrees.

In order to reduce the AC loss of the cable, another study was accomplished. According to this study:

- The AC loss in the improved design of the HTS cable is 0.356 times of the AC loss in the preliminary design.

- The AC loss could be further decreased if the reduction step of the twisting angle is selected to be smaller, but we would end up needing a much better computer system to handle the high computation cost.

\section{REFERENCES}

[1] J. Chigvinadze, V. Tavkhelidze, G. Mamniashvili, G. Donadze, J. V. Acrivos, and D. Gulamova, "Vibrating Reed Study of Superconducting Cuprates Fabricated by Superfast Melt Quenching in a Solar Furnace," Engineering, Technology \& Applied Science Research, vol. 9, no. 4, pp. 4495-4499, Aug. 2019, https://doi.org/10.48084/etasr.2948.

[2] M. Yazdani-Asrami, W. Song, X. Pei, M. Zhang, and W. Yuan, "AC Loss Characterization of HTS Pancake and Solenoid Coils Carrying Nonsinusoidal Currents," IEEE Transactions on Applied Superconductivity, vol. 30, no. 5, pp. 1-9, Aug. 2020, https://doi.org/ 10.1109/TASC.2020.2971840.

[3] M. Yazdani-Asrami, W. Song, M. Zhang, W. Yuan, and X. Pei, "Magnetization Loss in HTS Coated Conductor Exposed to Harmonic External Magnetic Fields for Superconducting Rotating Machine Applications," IEEE Access, vol. 9, pp. 77930-77937, 2021, https://doi.org/10.1109/ACCESS.2021.3062278.

[4] M. Yazdani-Asrami, M. Zhang, and W. Yuan, "Challenges for developing high temperature superconducting ring magnets for rotating electric machine applications in future electric aircrafts," Journal of Magnetism and Magnetic Materials, vol. 522, Mar. 2021, Art. no. 167543, https://doi.org/10.1016/j.jmmm.2020.167543.

[5] M. Yazdani-Asrami et al., "Fault current limiting HTS transformer with extended fault withstand time," Superconductor Science and Technology, vol. 32, no. 3, Feb. 2019, Art. no. 035006, https://doi.org/ $10.1088 / 1361-6668 /$ aaf7a8.
[6] W. Song et al., "AC Losses in Noninductive SFCL Solenoidal Coils Wound by Parallel Conductors," IEEE Transactions on Applied Superconductivity, vol. 30, no. 8, pp. 1-9, Dec. 2020, https://doi.org/ 10.1109/TASC.2020.3021339.

[7] D. I. Doukas, "Superconducting Transmission Systems: Review, Classification, and Technology Readiness Assessment," IEEE Transactions on Applied Superconductivity, vol. 29, no. 5, pp. 1-5, Aug. 2019, https://doi.org/10.1109/TASC.2019.2895395.

[8] G. Vyas and R. S. Dondapati, "AC losses in the development of superconducting magnetic energy storage devices," Journal of Energy Storage, vol. 27, Feb. 2020, Art. no. 101073, https://doi.org/10.1016/ j.est.2019.101073.

[9] H. Maeda and Y. Yanagisawa, "Recent Developments in HighTemperature Superconducting Magnet Technology (Review)," IEEE Transactions on Applied Superconductivity, vol. 24, no. 3, pp. 1-12, Jun. 2014, https://doi.org/10.1109/TASC.2013.2287707.

[10] H. Thomas, A. Marian, A. Chervyakov, S. Stückrad, D. Salmieri, and C. Rubbia, "Superconducting transmission lines - Sustainable electric energy transfer with higher public acceptance?," Renewable and Sustainable Energy Reviews, vol. 55, pp. 59-72, Mar. 2016, https://doi.org/10.1016/j.rser.2015.10.041.

[11] A. Zebar and L. Madani, "SFCL-SMES Control for Power System Transient Stability Enhancement Including SCIG-based Wind Generators," Engineering, Technology \& Applied Science Research, vol. 10, no. 2, pp. 5477-5482, Apr. 2020, https://doi.org/10.48084/ etasr. 3422 .

[12] A. Safaei, S. H. Hosseinian, and H. A. Abyaneh, "Enhancing the HVRT and LVRT Capabilities of DFIG-based Wind Turbine in an Islanded Microgrid," Engineering, Technology \& Applied Science Research, vol. 7, no. 6, pp. $2118-2123$, Dec. 2017, https://doi.org/10.48084/etasr.1541.

[13] J. X. Jin, X. Y. Chen, R. Qu, H. Y. Fang, and Y. Xin, "An integrated low-voltage rated HTS DC power system with multifunctions to suit smart grids," Physica C: Superconductivity and its Applications, vol. 510, pp. 48-53, Mar. 2015, https://doi.org/10.1016/j.physc.2015.01.006.

[14] T. Nakayama, T. Yagai, M. Tsuda, and T. Hamajima, "Micro Power Grid System With SMES and Superconducting Cable Modules Cooled by Liquid Hydrogen," IEEE Transactions on Applied Superconductivity, vol. 19, no. 3, pp. 2062-2065, Jun. 2009, https://doi.org/10.1109/ TASC.2009.2018743.

[15] M. Yazdani-Asrami, S. A. Gholamian, S. M. Mirimani, and J. Adabi, "Investigation on Effect of Magnetic Field Dependency Coefficient of Critical Current Density on the AC Magnetizing Loss in HTS Tapes Exposed to External Field," Journal of Superconductivity and Novel Magnetism, vol. 31, no. 12, pp. 3899-3910, Dec. 2018, https://doi.org/ 10.1007/s10948-018-4664-1.

[16] W. Chen et al., "Numerical study on critical current and AC loss for different structured stack slotted-core HTS cables," Cryogenics, vol. 103, Oct. 2019, Art. no. 102972, https://doi.org/10.1016/j.cryogenics. 2019.102972.

[17] M. Tsuda, J. Fujimoto, N. Harada, and T. Hamajima, "AC loss reduction of coaxial multi-layer HTS cable," IEEE Transactions on Applied Superconductivity, vol. 14, no. 2, pp. 642-645, Jun. 2004, https://doi.org/10.1109/TASC.2004.830014.

[18] M. Yazdani-Asrami, M. Staines, G. Sidorov, and A. Eicher, "Heat transfer and recovery performance enhancement of metal and superconducting tapes under high current pulses for improving fault current-limiting behavior of HTS transformers," Superconductor Science and Technology, vol. 33, no. 9, Aug. 2020, Art. no. 095014, https://doi.org/10.1088/1361-6668/aba542.

[19] J. Ogawa, S. Fukui, M. Sugai, S. Aoyama, N. Koseki, and T. Matsubara, "AC Loss in Each Layer of Three-Layer Twisted HTS Cable," IEEE Transactions on Applied Superconductivity, vol. 29, no. 5, pp. 1-6, Aug. 2019, https://doi.org/10.1109/TASC.2019.2901213.

[20] S. Fukui, R. Kojima, J. Ogawa, M. Yamaguchi, T. Sato, and O. Tsukamoto, "Numerical Analysis of AC Loss Characteristics of Cable Conductor Assembled by HTS Tapes in Polygonal Arrangement," IEEE Transactions on Applied Superconductivity, vol. 16, no. 2, pp. 143-146, Jun. 2006, https://doi.org/10.1109/TASC.2006.870818. 
[21] F. Grilli, S. Stavrev, B. Dutoit, and S. Spreafico, "Numerical analysis of the effects of the magnetic self-field on the transport properties of a multilayer HTS cable," IEEE Transactions on Applied Superconductivity, vol. 14, no. 1, pp. 94-102, Mar. 2004, https://doi.org/ 10.1109/TASC.2004.824333.

[22] H. Noji, S. Ooyama, and K. Nakajima, "Effective helical-pitch adjustment in a high-Tc superconducting cable for reducing AC losses," Physica C: Superconductivity, vol. 412-414, pp. 1206-1211, Oct. 2004, https://doi.org/10.1016/j.physc.2004.01.140.

[23] J. Zhu, X. Bao, L. Guo, Z. Xia, M. Qiu, and W. Yuan, "Optimal Design of Current Sharing in Transmission Conductors of a $110 \mathrm{kV} / 3 \mathrm{kA}$ Cold Dielectric Superconducting Cable Consisted of YBCO Tapes," IEEE Transactions on Applied Superconductivity, vol. 23, no. 3, pp. 54025055402505, Jun. 2013, https://doi.org/10.1109/TASC.2013.2244156.

[24] X. Liu, S. Wang, J. Qiu, J. G. Zhu, Y. Guo, and Z. W. Lin, "Robust Optimization in HTS Cable Based on Design for Six Sigma," IEEE Transactions on Magnetics, vol. 44, no. 6, pp. 978-981, Jun. 2008, https://doi.org/10.1109/TMAG.2007.916279.

[25] T.-T. Nguyen et al., "A Simplified Model of Coaxial, Multilayer HighTemperature Superconducting Power Cables with $\mathrm{Cu}$ Formers for Transient Studies," Energies, vol. 12, no. 8, Jan. 2019, Art. no. 1514, https://doi.org/10.3390/en12081514.

[26] S.-K. Ha et al., "Development of an Impedance Matching Program for Balancing the Current Distribution in a Tri-axial HTS Power Cable," Journal of Superconductivity and Novel Magnetism, vol. 26, no. 4, pp. 759-762, Apr. 2013, https://doi.org/10.1007/s10948-012-2012-4.

[27] A. Sadeghi, S. M. Seyyedbarzegar, and M. Yazdani-Asrami, "Transient analysis of a $22.9 \mathrm{kV} / 2 \mathrm{kA}$ HTS cable under short circuit using equivalent circuit model considering different fault parameters," Physica C: Superconductivity and its Applications, vol. 589, Oct. 2021, Art. no. 1353935, https://doi.org/10.1016/j.physc.2021.1353935.

[28] T. Hamajima et al., "Balanced three-phase distribution experiment of a triaxial HTS cable," Electronics and Communications in Japan, vol. 94, no. 2, pp. 51-58, 2011, https://doi.org/10.1002/ecj.10283.

[29] K. Wang, W. Ta, and Y. Gao, "The winding mechanical behavior of conductor on round core cables," Physica C: Superconductivity and its Applications, vol. 553, pp. 65-71, Oct. 2018, https://doi.org/10.1016/ j.physc.2018.08.012.

[30] J. Lee and G. Cha, "Magnetization loss calculation in superconducting power transmission cable," Cryogenics, vol. 41, no. 3, pp. 157-161, Mar. 2001, https://doi.org/10.1016/S0011-2275(01)00067-4.

[31] M. Yazdani-Asrami, S. A. Gholamian, S. M. Mirimani, and J. Adabi, "Experimental investigation for power loss measurement of superconducting coils under harmonic supply current," Measurement, vol. 132, pp. 324-329, Jan. 2019, https://doi.org/10.1016/j.measurement. 2018.03.042.

[32] M. Yazdani-Asrami, S. A. Gholamian, S. M. Mirimani, and J. Adabi, "Calculation of AC Magnetizing Loss of ReBCO Superconducting Tapes Subjected to Applied Distorted Magnetic Fields," Journal of Superconductivity and Novel Magnetism, vol. 31, no. 12, pp. 3875-3888, Dec. 2018, https://doi.org/10.1007/s10948-018-4695-7.

[33] A. Sadeghi and S. Seyyedbarzegar, "An accurate model of the hightemperature superconducting cable by using stochastic methods," Transformers Magazine, vol. 8, no. S5, pp. 70-76, Aug. 2021.

[34] J. Fleiter, M. Sitko, and A. Ballarino, "Analytical Formulation of $I_{c}$ Dependence on Torsion of YBCO and BSCCO Conductors," IEEE Transactions on Applied Superconductivity, vol. 23, no. 3, pp. 8000204 8000204, Jun. 2013, https://doi.org/10.1109/TASC.2012.2228292. 